\title{
EVALUATION OF VARIETIES OF GARDEN PEA (PISUM SATIVUM L.) WITH TIME OF SOWING IN NORTH GUJARAT CONDITIONS
}

\author{
S.K. ACHARYA ${ }^{1 *}$, G. S. PATEL ${ }^{2}$, J. R. VADODARIA ${ }^{3} \&$ MUKESH KUMAR $^{4}$ \\ ${ }^{1,4}$ Assistant Professor, College of Horticulture, SDAU, Jagudan Dist. Mehsana, India \\ ${ }^{2,3}$ Associate Professor, College of Horticulture, SDAU, Jagudan Dist. Mehsana, India
}

\begin{abstract}
A field experiment was conducted during the winter season of 2016, 2017 and 2018 at Jagudan (Gujarat). The experiment was laid out in split plot design with treatment comprising three times of sowing, viz. $3^{\text {rd }}$ week of October $\left(D_{1}\right)$, $4^{\text {th }}$ week of October $\left(D_{2}\right)$ and $2^{\text {nd }}$ week of November $\left(D_{3}\right)$ in main plots and 14 different pea cultivars. Results (Pooled data) indicated that significantly minimum days (73.48) were noticed in treatment $D_{3}$ ( $2^{\text {nd }}$ week of November). However, in pooled data maximum days (91.49) was recorded in $D_{3}\left(2^{\text {nd }}\right.$ week of November) and was at par with treatment $D_{2}$ ( $4^{\text {th }}$ week of October) but both were superior to early sown $D_{1}$ ( $3^{\text {rd }}$ week of October). As far as variety is concern minimum days (67.89) taken for first picking and last picking (79.44) was recorded in variety Kashi Nandini. Maximum number of picking (3.44) with variety VRPMR 9, maximum length of pod (9.44 cm) with variety Arka Karthik, number (33.75) of pods/ plant in variety GDFP 1, number of seeds per pod (7.56) were recorded with variety Kashi Shakti and Arka Karthik. Though the maximum weight of pods/plant (58.46 g), yield of green pod/ha (135.54 q) and shelling percentage $(60.40 \%)$ were observed in Bonneville. However, maximum protein content was reported in Pusa Pragati.
\end{abstract}

KEYWORDS: Growth, Garden Pea, Sowing Time, Pod, Yield, Pisum Sativum Etc.

Received: Nov 30, 2020; Accepted: Dec 19, 2020; Published: Jan 04, 2021; Paper Id.: IJASRDEC20204

\section{INTRODUCTION}

Garden pea [Pisum sativum var. hortense (L)] is the prominent vegetable legume cultivated on an area of 540 thousand ha in India with a production of 5422 thousand tons as recorded during 2017-18 with of a productivity of around 10 tons/ha. (Anonymous, 2018) Among the garden pea producing states of India, Gujarat having the very negligible area particularly confined in Banaskantha and Sabarkantha district. The crop is cultivated for its tender and immature pods for use as vegetable and mature dry pods for use as a pulse. In both cases, seeds are separated and used as vegetable or pulse. Peas are highly nutritive and contain high content of digestible protein $(7.2 \mathrm{~g} /$ $100 \mathrm{~g})$, Carbohydrate (15.8 g), Vitamin-C (9 mg), phosphorus (139 mg) and minerals.

Garden Pea is the most important legume food of the world and liking of this vegetable is increasing day by day due to the health awareness among the masses and it provides fair amount nutrients as well as mineral elements. Cultivar performs differently under different agro-climatic conditions and even though affected by the sowing time which decides the suitable agro-climate required for the successful growth and development of the crops. Various cultivars of same species grown even in same environment often have yield differences. Yield and quality of crop are very complex characteristics depending on certain biological alignments between environment and heredity. Pea yield is highly affected by edapho-climatic factors such as soil, water stress, frost and high 
temperatures (Salter 1963). Delay in sowing beyond optimum time resulted in a progressive reduction in the potential yield of the crop (Green et al. 1985). A large number of varieties are known in garden pea which differs in their optimum sowing dates and period of maturity. Hence, the positive effect of environmental factors on growth and yield could be harnessed if the information on optimum time of sowing is made available (Moniruzzaman et al., 2007) along with a suitable variety. The characteristics of a cultivar as well as combination of traits differ according to climatic conditions of the localities. Find out of that some of the national varieties performed very well under north Gujarat condition. Looking these facts in mind present experiment were undertaken -

\section{MATERIAL AND METHODS}

The experiment was carried out at College Farm, College of Horticulture, S. D. Agricultural University, Jagudan, Gujarat during three consecutive years from 2016 to 2018 in winter season. Experiment was laid out in Split Plot Design with four replications. Treatments comprised two factors viz., (main plot treatment) -time of sowing (d) with three levels viz.,3rd week of October $\left(d_{1}\right)$, 4th week of October $\left(d_{2}\right)$ and 2 nd week of November $\left(d_{3}\right)$ and (sub plot treatments)-varieties (v) fourteen varieties viz., Bonneville $\left(\mathrm{v}_{1}\right)$, Arkel, Pusa $\left(\mathrm{v}_{2}\right)$, Pragti $\left(\mathrm{v}_{3}\right)$, Azad P $1\left(\mathrm{v}_{4}\right)$, Kashi Nandini $\left(\mathrm{v}_{5}\right)$, Kashi Udai $\left(\mathrm{v}_{6}\right)$, Kashi Shakti ( $\left.\mathrm{v}_{7}\right)$, Arka Karthik ( $\left.\mathrm{v}_{8}\right)$, Lincoln ( $\left.\mathrm{v}_{9}\right)$, Early Giant ( $\left.\mathrm{v}_{10}\right)$, Azad P $3\left(\mathrm{v}_{11}\right)$, Kashi Mukti $\left(\mathrm{v}_{12}\right)$, VRPMR $9\left(\mathrm{v}_{13}\right)$ and GDFP $1\left(\mathrm{v}_{14}\right)$. Thus, making total forty two treatment combinations. Standard package of practices (Singh et al., 2004) were followed during the entire crop period. Observations were recorded from randomly selected ten tagged plants on growth, yield and quality traits and they were subjected to statistical analysis as per the standard procedure (Gomez and Gomez, 1984).

\section{RESULTS AND DISCUSSION}

Effect of various time of sowing on days taken for germination showed non-significant differences in individual years as well as pooled analysis. Among varieties, statistically significant minimum days taken to germination during 2016 (5.25), 2017 (5.50), 2018 (5.92) and in pooled data (5.56) were observed in variety Pusa Pragati $\left(\mathrm{V}_{3}\right)$ which was at par with varieties Kashi Nandini $\left(\mathrm{V}_{5}\right)$, Arka Karthi $\left(\mathrm{V}_{8}\right)$, Lincoln $\left(\mathrm{V}_{9}\right)$, Azad P $3\left(\mathrm{~V}_{11}\right)$ and Arkel $\left(\mathrm{V}_{2}\right)$ in individual year and pooled analysis but significantly superior over rest of the varieties. Germination percentage showed significant difference among the different varieties in individual year, but it was not significant in pooled analysis. However, maximum germination (\%) was recorded in variety Early Giant in 2016 (93.08\%) and 2018 (91.92\%). Though the maximum germination (83.92\%) during 2017 in Pusa Pragati. $\quad$ Significantly, minimum days taken for first flowering (47.93) was observed in $\mathrm{D}_{3}\left(2^{\text {nd }}\right.$ week of November) during pooled analysis. Among varieties there was significant difference for days taken to first flower among the varieties. The minimum day taken for flowering (44.08) was found in variety Kashi Nandini $\left(\mathrm{V}_{5}\right)$ in pooled data which was at par with variety Arkel $\left(\mathrm{V}_{2}\right)$, Pusa Pragati $\left(\mathrm{V}_{3}\right)$, Azad P $3\left(\mathrm{~V}_{11}\right)$ and Kashi Mukti $\left(\mathrm{V}_{12}\right)$.

Among time of sowing, minimum days (76.75) taken to first picking which showed earliness in treatment $\mathrm{D}_{3}\left(2^{\text {nd }}\right.$ week of November) whereas, in second and third year non-significant results were observed. In pooled analysis significantly minimum days (73.48) was noticed in treatment $\mathrm{D}_{3}$ ( $2^{\text {nd }}$ week of November). Minimum days (67.42) taken for first picking (Table 2) was recorded in variety Kashi Nandini (VRP-5) $\left(\mathrm{V}_{5}\right)$ in 2016, 2018 (66.25) and pooled (67.89) also. Non-significant results in individual year for days taken to last picking for time of sowing. However, in pooled data minimum days (91.49) was recorded in $\mathrm{D}_{3}$ ( $2^{\text {nd }}$ week of November) and was at par with treatment $\mathrm{D}_{2}$ ( $4^{\text {th }}$ week of October) but both were superior to early sown crop $\mathrm{D}_{1}\left(3^{\text {rd }}\right.$ week of October). 
Minimum days taken for last picking (79.44) was recorded with variety Kashi Nandini $\left(\mathrm{V}_{5}\right)$ and it was remain at par with variety Kashi Uday $\left(\mathrm{V}_{6}\right)$, Arkel $\left(\mathrm{V}_{2}\right)$ and Pusa Pragati $\left(\mathrm{V}_{3}\right)$ in pooled data. Maximum number (3.66) of picking was observed in $\mathrm{D}_{1}\left(3^{\text {rd }}\right.$ week of October) in $1^{\text {st }}$ year. However, rest of the year as well as pooled data showed nonsignificant results. Number of picking was also showed significant difference among the varieties during all the year and in pooled data (Table 3). Maximum number of picking (3.44) observed with variety VRPMR-9 ( $\left.\mathrm{V}_{13}\right)$. This indicates that the growth stage of early sown crop was more advanced than the late sown crops. Knott and Belcher (1998) and Sharma et al. (2014) also found advanced growth in October sown crop than the later sowings. Significantly maximum length of pod $(9.44 \mathrm{~cm})$ recorded with variety Arka Karthik $\left(\mathrm{V}_{8}\right)$. Maximum number (33.75) of pods/ plant was observed in variety GDFP $1\left(\mathrm{~V}_{14}\right)$ in $1^{\text {st }}$ year and subsequent year and pooled (29.44) data also.

Significant difference among the varieties for number of seeds/pod in individual year as well as pooled data. Maximum number of seeds per pod (7.56) was found with variety Kashi Shakti (VRP -7) ( $\left.\mathrm{V}_{7}\right)$ and Arka Karthik $\left(\mathrm{V}_{8}\right)$ which was statistically at par with variety $\mathrm{V}_{5}, \mathrm{~V}_{11}, \mathrm{~V}_{1}$ and $\mathrm{V}_{9}$. Maximum average weight of pods/plant (58.46 g) were observed in variety $\mathrm{V}_{1}$ (Bonneville) in $3^{\text {rd }}$ year and similar trends were noticed in $1^{\text {st }}$ year $(58.09 \mathrm{~g}), 2^{\text {nd }}$ year $(45.81 \mathrm{~g})$ and pooled (54.12 g) also.

Maximum yield of green pod/ha (135.54 q) was recorded in variety Bonneville $\left(\mathrm{V}_{1}\right)$ during course of investigation and in pooled analysis also. Performance of pea crop sown during October and November sown pea does not affects the yield and quality of garden pea; it might be due to the mild winter situation during the crop period over the years. The reduction in yield of different varieties with each delay in sowing might be due to high temperature which induced flowering before the plants had grown sufficiently to bear a good crop (Sidique et al. 2002). Similar results were also reported by Sharma et al. (2011), Sharma et al. (2014) Bozogluet al. (2007) Chadha et al. (2013), Chadha (2015), Futuless and Bake (2010), Kalloo et al. (2005), Khan et al. (2013) and Khichi et al. (2016). Maximum shelling percentage (60.40\%) were observed in variety Bonneville $\left(\mathrm{V}_{1}\right)$ in $3^{\text {rd }}$ year and similar trends were noticed in $1^{\text {st }}$ year $(60.13 \%), 2^{\text {nd }}$ year $(59.13$ $\%)$ and pooled $(59.88 \%)$ also.

Maximum protein content was reported with variety Pusa Pragati $\left(\mathrm{V}_{3}\right)$ during the course of study as well as in pooled analysis also. Variety $\mathrm{V}_{2}, \mathrm{~V}_{6}$ and $\mathrm{V}_{1}$ remain statistically at par with variety $\mathrm{V}_{3}$ in pooled analysis (Table 7). The results are in conformity with the finding of Sirwaiya, et al. (2018), Singh and Singh (2011).

\section{Interaction Effect of Different Date of Sowing with Varieties on Growth, Yield and Quality of Garden Pea}

Interaction effect of different date of sowing with varieties was found non-significant for all the growth, yield and quality parameters of garden pea.

\section{Economics of Varieties with Time of Sowing}

There were no significant differences observed for different time of sowing on yield of green pod and net return. Among the varieties, maximum net realization of $₹$ 211035/- per ha and highest B:C Ratio (4.11) were obtained with the variety Bonneville $\left(\mathrm{V}_{1}\right)$ followed by variety Azad P3 $\left(\mathrm{V}_{11}\right)$. Variation in the yield as well as BC ratio is might be due to the genetic response of the germplasm in a specific environment as well as edapho-climatic conditions suitable for that particular germplasm. The similar results observed by Sharma (2002) and Bilashini Devi et al. (2018). 
Table 1: Effect of Different Date of Sowing and Varieties on Days Taken for Germination and Germination (\%)

\begin{tabular}{|c|c|c|c|c|c|c|c|c|}
\hline \multirow[t]{2}{*}{ Treatment } & \multicolumn{4}{|c|}{ Days Taken for Germination } & \multicolumn{4}{|c|}{ Germination (\%) } \\
\hline & 2016 & 2017 & 2018 & Pooled & 2016 & 2017 & 2018 & Pooled \\
\hline \multicolumn{9}{|c|}{ Date of Sowing (Main Plot) } \\
\hline $\mathrm{D}_{1}$ & 6.59 & 6.43 & 6.93 & 6.65 & 83.82 & 80.48 & 83.82 & 82.71 \\
\hline $\mathrm{D}_{2}$ & 6.16 & 6.21 & 6.61 & 6.33 & 84.13 & 80.52 & 82.93 & 82.52 \\
\hline $\mathrm{D}_{3}$ & 6.29 & 6.21 & 6.55 & 6.35 & 83.80 & 78.63 & 83.13 & 81.85 \\
\hline S.Em. \pm & 0.24 & 0.14 & 0.13 & 0.10 & 0.53 & 0.78 & 0.4 & 0.34 \\
\hline C.D. at $5 \%$ & NS & NS & NS & NS & NS & NS & NS & NS \\
\hline C.V.\% & 28.80 & 16.35 & 14.65 & 20.74 & 4.70 & 7.40 & 3.60 & 5.39 \\
\hline \multicolumn{9}{|l|}{ Varieties (Sub Plot) } \\
\hline (Bonneville) $\mathrm{V}_{1}$ & 8.17 & 7.58 & 7.92 & 7.89 & 77.08 & 78.17 & 76.92 & 77.39 \\
\hline (Arkel) $\mathrm{V}_{2}$ & 5.83 & 6.42 & 6.08 & 6.11 & 82.50 & 82.83 & 82.58 & 82.64 \\
\hline (Pusa Pragati) $V_{3}$ & 5.25 & 5.50 & 5.92 & 5.56 & 80.00 & 83.92 & 79.67 & 81.19 \\
\hline$($ Azad P 1) V 4 & 6.58 & 5.50 & 6.83 & 6.31 & 86.33 & 82.50 & 86.00 & 84.94 \\
\hline (Kashi Nandini) $V_{5}$ & 5.25 & 5.83 & 6.42 & 5.83 & 85.50 & 80.75 & 84.42 & 83.56 \\
\hline (Kashi Udai) $V_{6}$ & 6.25 & 6.50 & 6.58 & 6.44 & 84.33 & 82.75 & 84.17 & 83.75 \\
\hline (Kashi Shakti) $V_{7}$ & 7.00 & 6.92 & 6.92 & 6.94 & 83.17 & 83.58 & 82.42 & 83.06 \\
\hline (Arka Karthik) $V_{8}$ & 5.42 & 6.25 & 5.92 & 5.86 & 79.17 & 79.33 & 77.67 & 78.72 \\
\hline (Lincoln) $\mathrm{V}_{9}$ & 6.33 & 5.92 & 6.08 & 6.11 & 90.42 & 77.75 & 88.17 & 85.44 \\
\hline (Early Giant) $\mathrm{V}_{10}$ & 6.33 & 6.50 & 7.42 & 6.75 & 93.08 & 60.67 & 91.92 & 81.89 \\
\hline$\left(\right.$ Azad P 3) $V_{11}$ & 5.83 & 5.58 & 6.50 & 5.97 & 84.17 & 78.33 & 84.33 & 82.28 \\
\hline (Kashi Mukti) $V_{12}$ & 7.00 & 6.50 & 6.67 & 6.72 & 78.50 & 80.67 & 77.58 & 78.92 \\
\hline (VRPMR 9) $V_{13}$ & 7.42 & 6.67 & 7.92 & 7.33 & 87.00 & 83.67 & 85.33 & 85.33 \\
\hline (GDFP 1) $V_{14}$ & 6.17 & 6.33 & 6.58 & 6.36 & 83.58 & 83.33 & 84.92 & 83.94 \\
\hline S.Em. \pm & 0.25 & 0.22 & 0.20 & 0.21 & 0.68 & 0.79 & 0.62 & 3.01 \\
\hline C.D. at $5 \%$ & 0.70 & 0.61 & 0.55 & 0.62 & 1.89 & 2.21 & 1.73 & NS \\
\hline \multicolumn{9}{|l|}{ Interaction } \\
\hline D x V & NS & NS & $\mathrm{NS}$ & NS & $\mathrm{NS}$ & 3.82 & 2.99 & $\mathrm{NS}$ \\
\hline C.V. \% & 13.70 & 11.96 & 10.22 & 11.99 & 2.79 & 3.42 & 2.57 & 2.93 \\
\hline
\end{tabular}

Table 2: Effect of Different Date of Sowing and Varieties on Days Taken for First Flowering and Days Taken for First Picking

\begin{tabular}{|c|c|c|c|c|c|c|c|c|}
\hline \multirow[t]{2}{*}{ Treatment } & \multicolumn{4}{|c|}{ Days Taken for First Flowering } & \multicolumn{4}{|c|}{ Days Taken for First Picking } \\
\hline & 2016 & 2017 & 2018 & Pooled & 2016 & 2017 & 2018 & Pooled \\
\hline \multicolumn{9}{|c|}{ Date of Sowing (Main Plot) } \\
\hline $\mathrm{D}_{1}$ & 50.68 & 50.48 & 50.70 & 50.62 & 80.82 & 73.43 & 79.84 & 78.03 \\
\hline $\mathrm{D}_{2}$ & 48.75 & 49.07 & 40.34 & 48.72 & 78.00 & 72.91 & 77.54 & 76.15 \\
\hline $\mathrm{D}_{3}$ & 47.75 & 48.16 & 47.88 & 47.93 & 76.75 & 70.61 & 75.68 & 73.48 \\
\hline S.Em.土 & 0.44 & 0.49 & 0.12 & 0.23 & 0.51 & 1.42 & 0.27 & 0.51 \\
\hline C.D. at $5 \%$ & 1.53 & 1.71 & 0.42 & 0.67 & 1.78 & $\mathrm{NS}$ & 0.95 & 1.52 \\
\hline C.V. \% & 6.75 & 7.52 & 1.86 & 5.94 & 4.89 & 14.89 & 2.67 & 8.75 \\
\hline \multicolumn{9}{|l|}{ Varieties (Sub Plot) } \\
\hline (Bonneville) $\mathrm{V}_{1}$ & 50.25 & 49.50 & 50.25 & 50.00 & 94.25 & 78.42 & 93.83 & 88.83 \\
\hline (Arkel) $\mathrm{V}_{2}$ & 44.75 & 47.25 & 44.83 & 45.61 & 70.75 & 72.08 & 70.58 & 71.14 \\
\hline (Pusa Pragati) $V_{3}$ & 45.00 & 44.67 & 45.58 & 45.08 & 71.25 & 73.33 & 72.58 & 72.39 \\
\hline$\left(\right.$ Azad P 1) $V_{4}$ & 52.75 & 52.50 & 53.00 & 52.75 & 82.50 & 80.75 & 80.67 & 79.19 \\
\hline (Kashi Nandini) $V_{5}$ & 43.25 & 45.50 & 43.50 & 44.08 & 67.42 & 70.00 & 66.25 & 67.89 \\
\hline (Kashi Udai) $\mathrm{V}_{6}$ & 45.33 & 45.33 & 45.58 & 45.42 & 69.17 & 69.00 & 68.08 & 68.75 \\
\hline (Kashi Shakti) $V_{7}$ & 52.08 & 52.08 & 50.83 & 51.67 & 80.92 & 71.00 & 80.83 & 77.58 \\
\hline (Arka Karthik) $\mathrm{V}_{8}$ & 47.92 & 48.58 & 47.75 & 48.08 & 75.08 & 71.75 & 74.58 & 71.66 \\
\hline (Lincoln) $\mathrm{V}_{9}$ & 58.00 & 55.92 & 54.25 & 56.06 & 88.33 & 73.67 & 85.75 & 82.58 \\
\hline (Early Giant) $\mathrm{V}_{10}$ & 44.67 & 48.58 & 45.92 & 46.39 & 94.58 & 67.58 & 93.33 & 85.17 \\
\hline$\left(\right.$ Azad P 3) $V_{11}$ & 45.00 & 45.33 & 45.08 & 45.14 & 72.33 & 67.92 & 72.42 & 70.89 \\
\hline (Kashi Mukti) $V_{12}$ & 45.17 & 45.92 & 46.08 & 45.72 & 72.50 & 72.42 & 72.17 & 72.36 \\
\hline (VRPMR 9) $V_{13}$ & 60.83 & 56.42 & 61.75 & 59.67 & 79.00 & 73.17 & 77.58 & 76.58 \\
\hline (GDFP 1) $V_{14}$ & 51.83 & 51.75 & 51.17 & 51.58 & 81.25 & 71.33 & 78.92 & 77.17 \\
\hline S.Em. \pm & 0.47 & 0.49 & 0.38 & 0.73 & 0.50 & 2.43 & 0.48 & 2.73 \\
\hline C.D. at $5 \%$ & 1.33 & 1.37 & 1.07 & 2.11 & 1.41 & $\mathrm{NS}$ & 1.34 & 7.95 \\
\hline \multicolumn{9}{|l|}{ Interaction } \\
\hline D x V & NS & 2.37 & 1.86 & NS & NS & $\mathrm{NS}$ & 2.32 & $\mathrm{NS}$ \\
\hline C.V.\% & 3.35 & 3.44 & 2.71 & 3.18 & 2.21 & 11.76 & 2.13 & 6.65 \\
\hline
\end{tabular}


Table 3: Effect of Different Date of Sowing and Varieties on Days Taken for Last Picking and Number of pickings

\begin{tabular}{|c|c|c|c|c|c|c|c|c|}
\hline \multirow[t]{2}{*}{ Treatment } & \multicolumn{4}{|c|}{ Days Taken for Last Picking } & \multicolumn{4}{|c|}{ Number of Pickings } \\
\hline & 2016 & 2017 & 2018 & Pooled & 2016 & 2017 & 2018 & Pooled \\
\hline \multicolumn{9}{|c|}{ Date of sowing (Main Plot) } \\
\hline $\mathrm{D}_{1}$ & 95.07 & 92.84 & 94.43 & 94.11 & 3.66 & 2.20 & 3.59 & 3.15 \\
\hline $\mathrm{D}_{2}$ & 93.14 & 91.36 & 93.09 & 92.53 & 3.25 & 2.30 & 3.25 & 2.93 \\
\hline $\mathrm{D}_{3}$ & 91.82 & 90.52 & 92.14 & 91.49 & 2.96 & 2.36 & 3.02 & 2.78 \\
\hline S.Em.土 & 0.74 & 0.73 & 0.58 & 0.40 & 0.15 & 0.09 & 0.13 & 0.14 \\
\hline C.D. at $5 \%$ & NS & NS & NS & 1.18 & 0.52 & NS & NS & NS \\
\hline C.V. \% & 5.96 & 5.93 & 4.66 & 5.55 & 34.31 & 28.97 & 30.88 & 32.37 \\
\hline \multicolumn{9}{|l|}{ Varieties (Sub Plot) } \\
\hline (Bonneville) $\mathrm{V}_{1}$ & 113.33 & 101.92 & 112.83 & 109.36 & 3.58 & 2.67 & 3.50 & 3.25 \\
\hline (Arkel) $\mathrm{V}_{2}$ & 80.00 & 81.00 & 79.92 & 80.31 & 2.83 & 2.33 & 2.83 & 2.67 \\
\hline (Pusa Pragati) $V_{3}$ & 84.00 & 83.42 & 83.50 & 83.64 & 3.58 & 2.25 & 3.58 & 3.14 \\
\hline$\left(\right.$ Azad P 1) $V_{4}$ & 100.25 & 96.58 & 98.00 & 98.28 & 3.75 & 2.50 & 3.67 & 3.31 \\
\hline (Kashi Nandini) $V_{5}$ & 77.25 & 85.08 & 76.00 & 79.44 & 2.83 & 2.17 & 2.83 & 2.61 \\
\hline (Kashi Udai) $\mathrm{V}_{6}$ & 80.25 & 79.83 & 79.50 & 79.86 & 3.42 & 2.58 & 3.33 & 3.11 \\
\hline (Kashi Shakti) $V_{7}$ & 91.75 & 88.67 & 90.50 & 90.31 & 3.75 & 2.58 & 3.67 & 3.33 \\
\hline (Arka Karthik) $\mathrm{V}_{8}$ & 92.50 & 91.50 & 92.25 & 92.08 & 2.83 & 2.00 & 3.08 & 2.64 \\
\hline (Lincoln) $\mathrm{V}_{9}$ & 101.75 & 101.42 & 100.67 & 101.28 & 3.75 & 2.42 & 3.75 & 3.31 \\
\hline (Early Giant) $\mathrm{V}_{10}$ & 103.25 & 101.50 & 104.33 & 103.03 & 2.33 & 1.50 & 2.33 & 2.06 \\
\hline$\left(\right.$ Azad P 3) $V_{11}$ & 93.92 & 93.58 & 94.33 & 93.94 & 3.75 & 2.50 & 3.75 & 3.33 \\
\hline (Kashi Mukti) $V_{12}$ & 83.17 & 85.50 & 87.08 & 85.25 & 3.08 & 2.17 & 3.08 & 2.78 \\
\hline (VRPMR 9) $V_{13}$ & 103.42 & 95.08 & 103.67 & 100.72 & 4.00 & 2.33 & 4.00 & 3.44 \\
\hline (GDFP 1) $V_{14}$ & 102.00 & 96.92 & 102.50 & 100.47 & 2.58 & 2.00 & 2.58 & 2.39 \\
\hline S.Em. \pm & 0.62 & 0.71 & 0.65 & 1.59 & 0.15 & 0.14 & 0.14 & 0.11 \\
\hline C.D. at $5 \%$ & 1.75 & 1.98 & 1.81 & 4.62 & 0.41 & 0.40 & 0.40 & 0.33 \\
\hline \multicolumn{9}{|l|}{ Interaction } \\
\hline D X V & NS & 3.46 & 3.14 & NS & NS & NS & NS & NS \\
\hline C.V. \% & 2.31 & 2.70 & 2.41 & 2.48 & 15.25 & 21.79 & 15.18 & 16.91 \\
\hline
\end{tabular}

Table 4: Effect of Different Date of Sowing and Varieties on Length of Pod (cm) and Number of Pods/Plant

\begin{tabular}{|c|c|c|c|c|c|c|c|c|}
\hline \multirow[t]{2}{*}{ Treatment } & \multicolumn{4}{|c|}{ Length of Pod $(\mathrm{cm})$} & \multicolumn{4}{|c|}{ Number of Pods/Plant } \\
\hline & 2016 & 2017 & 2018 & Pooled & 2016 & 2017 & 2018 & Pooled \\
\hline \multicolumn{9}{|c|}{ Date of Sowing (Main Plot) } \\
\hline $\mathrm{D}_{1}$ & 7.92 & 7.71 & 7.88 & 7.83 & 20.36 & 12.32 & 20.52 & 17.73 \\
\hline $\mathrm{D}_{2}$ & 7.94 & 7.66 & 7.94 & 7.85 & 21.50 & 12.71 & 21.20 & 18.47 \\
\hline $\mathrm{D}_{3}$ & 7.94 & 7.70 & 7.89 & 7.84 & 20.30 & 11.41 & 20.41 & 17.37 \\
\hline S.Em. \pm & 0.05 & 0.05 & 0.06 & 0.03 & 0.69 & 0.33 & 0.82 & 0.38 \\
\hline C.D. at $5 \%$ & NS & NS & NS & NS & NS & NS & NS & NS \\
\hline C.V. \% & 4.48 & 5.10 & 5.33 & 4.98 & 24.85 & 20.54 & 29.92 & 27.26 \\
\hline \multicolumn{9}{|l|}{ Varieties (Sub Plot) } \\
\hline (Bonneville) $\mathrm{V}_{1}$ & 8.11 & 7.71 & 8.06 & 7.93 & 26.08 & 17.25 & 26.17 & 23.17 \\
\hline (Arkel) $\mathrm{V}_{2}$ & 8.59 & 8.34 & 8.56 & 8.50 & 21.00 & 12.83 & 20.75 & 18.19 \\
\hline (Pusa Pragati) $V_{3}$ & 7.86 & 7.83 & 7.82 & 7.84 & 13.58 & 7.67 & 14.08 & 11.78 \\
\hline$\left(\right.$ Azad P 1) $V_{4}$ & 8.03 & 7.82 & 8.00 & 7.95 & 18.33 & 9.25 & 18.33 & 15.31 \\
\hline (Kashi Nandini) $V_{5}$ & 7.85 & 7.68 & 7.82 & 7.79 & 17.58 & 9.25 & 17.58 & 14.81 \\
\hline (Kashi Udai) $\mathrm{V}_{6}$ & 7.96 & 7.76 & 7.93 & 7.88 & 17.33 & 9.08 & 17.25 & 14.56 \\
\hline (Kashi Shakti) $V_{7}$ & 8.23 & 7.93 & 8.20 & 8.12 & 18.33 & 9.42 & 18.83 & 15.53 \\
\hline (Arka Karthik) $V_{8}$ & 9.63 & 9.09 & 9.60 & 9.44 & 17.92 & 9.83 & 18.08 & 15.28 \\
\hline$\left(\right.$ Lincoln) $\mathrm{V}_{9}$ & 7.23 & 7.36 & 7.20 & 7.26 & 19.75 & 10.83 & 19.58 & 16.67 \\
\hline (Early Giant) $V_{10}$ & 6.87 & 6.65 & 6.84 & 6.79 & 14.58 & 7.42 & 14.75 & 12.25 \\
\hline$\left(\right.$ Azad P 3) $V_{11}$ & 8.23 & 7.82 & 8.20 & 8.08 & 29.58 & 18.58 & 27.83 & 25.33 \\
\hline (Kashi Mukti) $V_{12}$ & 9.09 & 8.67 & 9.06 & 8.94 & 15.08 & 10.58 & 15.42 & 13.69 \\
\hline$\left(\right.$ VRPMR 9) $V_{13}$ & 8.33 & 8.22 & 8.30 & 8.28 & 27.33 & 17.25 & 27.50 & 24.03 \\
\hline (GDFP 1) V 14 & 5.05 & 4.80 & 5.01 & 4.95 & 33.75 & 20.83 & 33.75 & 29.44 \\
\hline S.Em. \pm & 0.05 & 0.06 & 0.05 & 0.06 & 0.47 & 0.43 & 0.78 & 0.66 \\
\hline C.D. at $5 \%$ & 0.13 & 0.15 & 0.13 & 0.17 & 1.31 & 1.20 & 1.40 & 1.92 \\
\hline \multicolumn{9}{|l|}{ Interaction } \\
\hline D x V & NS & 0.26 & $\mathrm{NS}$ & NS & NS & 2.08 & 2.32 & NS \\
\hline C.V. \% & 1.98 & 2.47 & 1.99 & 2.15 & 7.81 & 12.25 & 8.00 & 8.90 \\
\hline
\end{tabular}


Table 5: Effect of Different Date of Sowing and Varieties on Number of Seeds/Pod and Average Weight of Pods/Plant (g)

\begin{tabular}{|c|c|c|c|c|c|c|c|c|}
\hline \multirow[t]{2}{*}{ Treatment } & \multicolumn{4}{|c|}{ Number of Seeds/Pod } & \multicolumn{4}{|c|}{ Average Weight of Pods/Plant (g) } \\
\hline & 2016 & 2017 & 2018 & Pooled & 2016 & 2017 & 2018 & Pooled \\
\hline \multicolumn{9}{|c|}{ Date of Sowing (Main Plot) } \\
\hline $\mathrm{D}_{1}$ & 6.96 & 5.96 & 6.95 & 6.63 & 48.13 & 36.63 & 48.43 & 44.40 \\
\hline $\mathrm{D}_{2}$ & 7.77 & 6.13 & 7.59 & 7.16 & 48.43 & 36.95 & 48.13 & 44.50 \\
\hline $\mathrm{D}_{3}$ & 6.98 & 6.30 & 7.13 & 6.80 & 47.40 & 38.12 & 47.49 & 44.34 \\
\hline S.Em. \pm & 0.11 & 0.06 & 0.13 & 0.15 & 0.66 & 0.46 & 0.47 & 0.31 \\
\hline C.D. at $5 \%$ & 0.38 & 0.21 & 0.46 & $\mathrm{NS}$ & NS & NS & NS & NS \\
\hline C.V. \% & 11.46 & 7.30 & 13.74 & 11.51 & 10.35 & 9.16 & 7.30 & 9.06 \\
\hline \multicolumn{9}{|l|}{ Varieties (Sub Plot) } \\
\hline (Bonneville) $\mathrm{V}_{1}$ & 7.33 & 6.75 & 7.33 & 7.14 & 58.09 & 45.81 & 58.46 & 54.12 \\
\hline (Arkel) $\mathrm{V}_{2}$ & 7.08 & 6.17 & 7.33 & 6.86 & 48.90 & 39.88 & 50.42 & 46.40 \\
\hline (Pusa Pragati) $V_{3}$ & 6.75 & 6.00 & 6.58 & 6.44 & 40.11 & 33.79 & 40.83 & 38.24 \\
\hline$\left(\right.$ Azad P 1) $V_{4}$ & 6.67 & 5.67 & 6.75 & 6.36 & 54.32 & 40.76 & 54.80 & 49.96 \\
\hline (Kashi Nandini) $\mathrm{V}_{5}$ & 7.92 & 6.92 & 7.50 & 7.44 & 42.98 & 35.18 & 43.46 & 40.54 \\
\hline (Kashi Udai) $\mathrm{V}_{6}$ & 6.67 & 6.00 & 7.00 & 6.56 & 42.96 & 32.24 & 43.21 & 39.47 \\
\hline (Kashi Shakti) $V_{7}$ & 8.17 & 6.50 & 8.00 & 7.56 & 41.50 & 30.81 & 42.38 & 38.23 \\
\hline (Arka Karthik) $V_{8}$ & 7.92 & 6.83 & 7.92 & 7.56 & 43.21 & 31.28 & 42.50 & 39.00 \\
\hline (Lincoln) $\mathrm{V}_{9}$ & 7.42 & 6.25 & 7.67 & 7.11 & 46.04 & 33.13 & 45.42 & 41.53 \\
\hline (Early Giant) $\mathrm{V}_{10}$ & 7.25 & 5.67 & 7.25 & 6.72 & 51.29 & 37.93 & 51.50 & 46.91 \\
\hline$\left(\right.$ Azad P 3) $V_{11}$ & 8.17 & 6.00 & 8.00 & 7.39 & 55.79 & 41.97 & 54.00 & 50.59 \\
\hline (Kashi Mukti) $V_{12}$ & 7.42 & 6.33 & 7.50 & 7.08 & 42.96 & 35.91 & 43.50 & 40.79 \\
\hline (VRPMR 9) $V_{13}$ & 6.50 & 5.67 & 6.67 & 6.28 & 53.04 & 41.59 & 52.42 & 40.02 \\
\hline (GDFP 1) $\mathrm{V}_{14}$ & 6.08 & 5.08 & 5.58 & 5.58 & 50.63 & 40.99 & 49.33 & 46.98 \\
\hline S.Em. \pm & 0.22 & 0.18 & 0.19 & 0.16 & 0.54 & 0.70 & 0.53 & 0.81 \\
\hline C.D. at $5 \%$ & 0.61 & 0.50 & 0.52 & 0.45 & 1.50 & 1.95 & 1.47 & 2.35 \\
\hline \multicolumn{9}{|l|}{ Interaction } \\
\hline D $\times$ V & NS & 0.87 & NS & NS & NS & 3.38 & 2.55 & NS \\
\hline C.V. \% & 10.44 & 10.08 & 8.98 & 9.86 & 3.87 & 6.48 & 3.79 & 4.61 \\
\hline
\end{tabular}

Table 6: Effect of Different Date of Sowing and Varieties on Yield of Pods/Ha (q) and Shelling (\%)

\begin{tabular}{|c|c|c|c|c|c|c|c|c|}
\hline \multirow[t]{2}{*}{ Treatment } & \multicolumn{4}{|c|}{ Yield of pods/ha (q) } & \multicolumn{4}{|c|}{ Shelling (\%) } \\
\hline & 2016 & 2017 & 2018 & Pooled & 2016 & 2017 & 2018 & Pooled \\
\hline \multicolumn{9}{|c|}{ Date of sowing (Main Plot) } \\
\hline $\mathrm{D}_{1}$ & 112.30 & 98.91 & 111.39 & 107.53 & 52.23 & 52.71 & 52.05 & 52.33 \\
\hline $\mathrm{D}_{2}$ & 113.00 & 99.76 & 110.69 & 107.82 & 52.52 & 52.75 & 53.13 & 52.80 \\
\hline $\mathrm{D}_{3}$ & 110.60 & 102.92 & 109.23 & 107.58 & 52.21 & 52.69 & 52.77 & 52.56 \\
\hline S.Em. \pm & 1.55 & 1.23 & 1.08 & 0.75 & 0.67 & 0.615 & 0.76 & 0.40 \\
\hline C.D. at $5 \%$ & NS & NS & NS & NS & NS & NS & NS & NS \\
\hline C.V. \% & 10.35 & 9.16 & 7.30 & 9.04 & 9.60 & 8.72 & 10.81 & 9.75 \\
\hline \multicolumn{9}{|l|}{ Varieties (Sub Plot) } \\
\hline (Bonneville) $\mathrm{V}_{1}$ & 135.54 & 123.68 & 134.45 & 131.22 & 60.13 & 59.13 & 60.40 & 59.88 \\
\hline (Arkel) $\mathrm{V}_{2}$ & 114.11 & 107.67 & 115.96 & 112.58 & 52.81 & 54.10 & 53.29 & 53.40 \\
\hline (Pusa Pragati) $V_{3}$ & 93.59 & 91.24 & 93.92 & 92.91 & 49.38 & 49.67 & 49.81 & 49.62 \\
\hline$\left(\right.$ Azad P 1) $V_{4}$ & 126.74 & 110.05 & 126.04 & 120.95 & 56.31 & 54.83 & 56.60 & 55.92 \\
\hline (Kashi Nandini) $V_{5}$ & 100.29 & 94.98 & 99.95 & 98.41 & 45.21 & 47.42 & 45.65 & 46.09 \\
\hline (Kashi Udai) $\mathrm{V}_{6}$ & 100.24 & 87.04 & 99.38 & 95.55 & 43.40 & 44.35 & 43.77 & 43.84 \\
\hline (Kashi Shakti) $V_{7}$ & 96.79 & 83.19 & 97.46 & 92.49 & 54.96 & 53.50 & 55.23 & 54.56 \\
\hline (Arka Karthik) $\mathrm{V}_{8}$ & 100.82 & 84.45 & 97.75 & 94.34 & 57.65 & 58.08 & 57.94 & 57.89 \\
\hline (Lincoln) $\mathrm{V}_{9}$ & 107.43 & 89.44 & 104.46 & 100.44 & 54.15 & 55.25 & 54.44 & 54.61 \\
\hline (Early Giant) $\mathrm{V}_{10}$ & 119.68 & 102.40 & 118.45 & 113.51 & 55.40 & 55.83 & 55.69 & 55.64 \\
\hline (Azad P 3) $V_{11}$ & 130.18 & 113.32 & 124.20 & 122.57 & 46.90 & 48.83 & 47.19 & 47.64 \\
\hline (Kashi Mukti) $V_{12}$ & 100.24 & 96.96 & 100.05 & 99.08 & 47.65 & 48.50 & 47.94 & 48.03 \\
\hline (VRPMR 9) $V_{13}$ & 123.76 & 112.30 & 120.56 & 118.88 & 56.15 & 55.00 & 56.44 & 55.86 \\
\hline (GDFP 1) $\mathrm{V}_{14}$ & 118.13 & 110.67 & 113.47 & 114.09 & 52.40 & 53.56 & 52.69 & 52.88 \\
\hline S.Em. \pm & 1.25 & 1.88 & 1.21 & 1.82 & 0.53 & 0.52 & 054 & 0.40 \\
\hline C.D. at $5 \%$ & 3.50 & 5.28 & 3.38 & 5.29 & 1.48 & 1.52 & 1.50 & 1.17 \\
\hline \multicolumn{9}{|l|}{ Interaction } \\
\hline D x V & NS & 9.13 & 5.86 & NS & NS & 2.63 & NS & NS \\
\hline C.V. \% & 3.87 & 6.48 & 3.79 & 4.76 & 3.49 & 3.56 & 3.53 & 3.53 \\
\hline
\end{tabular}


Table 7: Effect of Different Date of Sowing and Varieties on Protein Content (\%)

\begin{tabular}{|c|c|c|c|c|}
\hline \multirow[t]{2}{*}{ Treatment } & \multicolumn{4}{|c|}{ Protein Content (\%) } \\
\hline & 2016 & 2017 & 2018 & Pooled \\
\hline \multicolumn{5}{|c|}{ Date of Sowing (Main Plot) } \\
\hline$\overline{D_{1}}$ & 22.97 & 22.92 & 22.92 & 22.94 \\
\hline $\mathrm{D}_{2}$ & 22.90 & 22.95 & 22.89 & 22.91 \\
\hline $\mathrm{D}_{3}$ & 22.53 & 22.94 & 22.73 & 22.73 \\
\hline S.Em. \pm & 0.185 & 0.04 & 0.185 & 0.09 \\
\hline C.D. at $5 \%$ & NS & NS & NS & NS \\
\hline C.V. \% & 6.08 & 1.33 & 6.07 & 5.01 \\
\hline \multicolumn{5}{|l|}{ Varieties (Sub Plot) } \\
\hline (Bonneville) $\mathrm{V}_{1}$ & 23.97 & 23.88 & 24.02 & 23.95 \\
\hline (Arkel) $\mathrm{V}_{2}$ & 23.97 & 24.09 & 24.02 & 24.03 \\
\hline (Pusa Pragati) $\mathrm{V}_{3}$ & 24.07 & 24.16 & 24.11 & 24.11 \\
\hline$\left(\right.$ Azad P 1) $V_{4}$ & 22.45 & 22.82 & 22.49 & 22.59 \\
\hline (Kashi Nandini) $V_{5}$ & 22.48 & 22.55 & 22.52 & 22.52 \\
\hline (Kashi Udai) $\mathrm{V}_{6}$ & 24.01 & 23.86 & 24.06 & 23.98 \\
\hline (Kashi Shakti) $V_{7}$ & 23.52 & 23.72 & 23.97 & 23.60 \\
\hline (Arka Karthik) $V_{8}$ & 22.66 & 22.96 & 22.70 & 22.77 \\
\hline (Lincoln) $\mathrm{V}_{9}$ & 23.00 & 23.02 & 23.04 & 23.02 \\
\hline (Early Giant) $\mathrm{V}_{10}$ & 22.63 & 22.78 & 22.67 & 22.69 \\
\hline$\left(\right.$ Azad P 3) $V_{11}$ & 21.54 & 21.92 & 21.59 & 21.69 \\
\hline (Kashi Mukti) $V_{12}$ & 22.30 & 22.26 & 22.34 & 22.30 \\
\hline$\left(\right.$ VRPMR 9) $V_{13}$ & 21.57 & 21.78 & 21.61 & 21.65 \\
\hline (GDFP 1) $V_{14}$ & 21.04 & 21.26 & 21.09 & 21.13 \\
\hline S.Em. \pm & 0.03 & 0.07 & 0.03 & 0.05 \\
\hline C.D. at $5 \%$ & 0.09 & 0.21 & 0.09 & 0.16 \\
\hline \multicolumn{5}{|l|}{ Interaction } \\
\hline D x V & NS & 0.36 & NS & NS \\
\hline C.V. \% & 0.50 & 1.11 & 0.50 & 0.76 \\
\hline
\end{tabular}

Table 8 Effect of Different Date of Sowing on Benefit: Cost Ratio

\begin{tabular}{|c|c|c|c|c|c|c|}
\hline No. & Treatments & $\begin{array}{c}\text { Yield per } \\
\text { Hectare }(q)\end{array}$ & $\begin{array}{c}\text { Gross } \\
\text { Income }(\bar{\Sigma})\end{array}$ & $\begin{array}{c}\text { Cost of } \\
\text { Cultivation }(\Sigma)\end{array}$ & Net Income (文) & $\begin{array}{l}\text { B:C } \\
\text { Ratio }\end{array}$ \\
\hline$D_{1}$ & $3^{\text {rd }}$ week of October & 107.53 & 215060 & 51405 & 163655 & 3.18 \\
\hline $\mathrm{D}_{2}$ & $4^{\text {th }}$ week of October & 107.82 & 215060 & 51405 & 164235 & 3.19 \\
\hline $\mathrm{D}_{3}$ & $2^{\text {nd }}$ week of November & 107.58 & 215060 & 51405 & 163755 & 3.19 \\
\hline
\end{tabular}

Table 9 Effect of Different Varieties on Benefit: Cost Ratio

\begin{tabular}{|c|c|c|c|c|c|c|}
\hline No. & Treatments & $\begin{array}{c}\text { Yield per } \\
\operatorname{Hectare}(q)\end{array}$ & 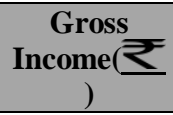 & $\begin{array}{c}\text { Cost of } \\
\text { Cultivation( }\end{array}$ & $\begin{array}{l}\text { Net } \\
\text { Income( } \\
\underline{\Sigma}\end{array}$ & B:C Ratio \\
\hline $\mathrm{V}_{1}$ & Bonneville & 131.22 & 262440 & 51405 & 211035 & 4.11 \\
\hline $\mathrm{V}_{2}$ & Arkel & 112.58 & 225160 & 51405 & 173755 & 3.38 \\
\hline $\mathrm{V}_{3}$ & Pusa Pragti & 92.91 & 185820 & 51405 & 134415 & 2.61 \\
\hline $\mathrm{V}_{4}$ & Azad P-1 & 120.95 & 241900 & 51405 & 190495 & 3.71 \\
\hline $\mathrm{V}_{6}$ & Kashi Udai $\quad$ (VRP -6) & 95.55 & 191100 & 51405 & 139695 & 2.72 \\
\hline $\mathrm{V}_{7}$ & Kashi Shakti (VRP -7) & 92.49 & 184980 & 51405 & 133575 & 2.60 \\
\hline $\mathrm{V}_{8}$ & Arka Karthik & 94.34 & 188680 & 51405 & 137275 & 2.67 \\
\hline $\mathrm{V}_{9}$ & Lincoln & 100.44 & 200880 & 51405 & 149475 & 2.91 \\
\hline $\mathrm{V}_{10}$ & Early Giant & 113.51 & 227020 & 51405 & 175615 & 3.42 \\
\hline $\mathrm{V}_{14}$ & GDFP-1 & 114.09 & 228180 & 51405 & 176775 & 3.44 \\
\hline
\end{tabular}




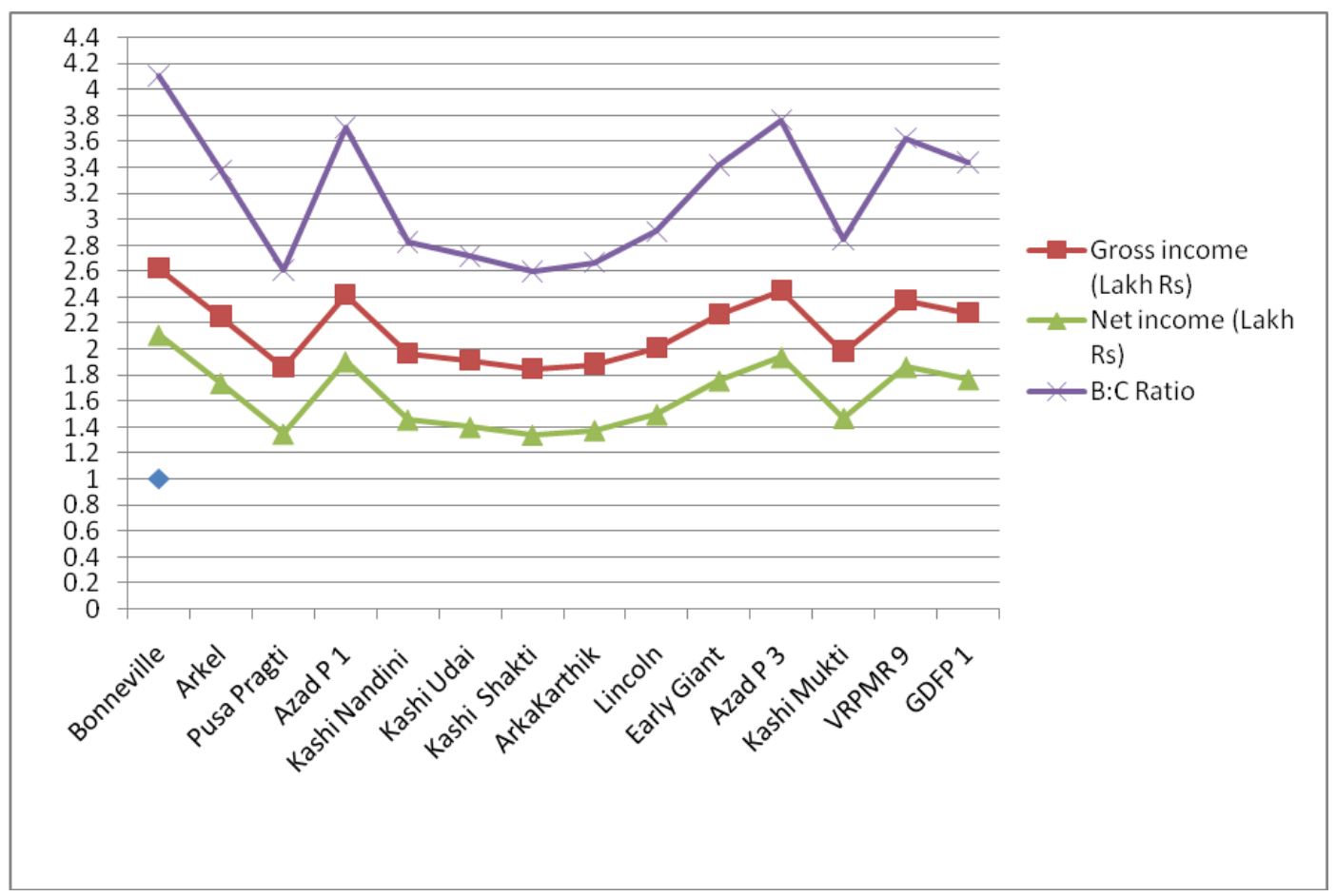

Figure 1: Effect of Different Varieties on Gross Income, Net Income and Benefit: Cost Ratio

\section{ACKNOWLEDGEMENTS}

This study was carried out with financial support from S.D. Agricultural University and Government of Gujarat, Gandhinagar, INDIA.

\section{REFERENCES}

1. Anonymous 2018. Horticulture Statistics at a Glance. National Horticulture Board, Gurugram.41p.

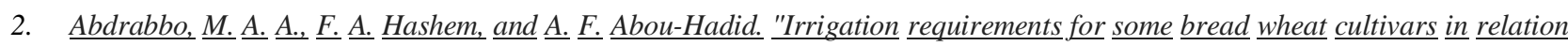
to planting dates." J. Agric. Sci. $\underline{\text { \& }} \underline{\operatorname{Res}} \underline{3.1}$ (2016): 23-40.

3. Bilashini Devi, Thoithoi Devi M M, Jha, A K, Yumnam, Anjoo, Balusamy, A, Verma, V K Talang, H D, Deshmukh, N A, Rymbai, H. and Assumi, S R. 2018. Yield and yield attributes of garden pea (Pisum sativum var. hortense L.) as influenced by nutrient management practices under agroclimatic conditions of Meghalaya. International Journal of Current Microbiology and Applied Sciences 7(09): 3447-3454.

4. Bozoglu, H, Peksen, E, Peksen, A and Gulumser, A. 2007. Determination of the yield performance and harvesting periods of fifteen pea (Pisum sativum L.) cultivars sown in autumn and spring. Pakistan Journal of Botany 39(6): 2017-2025.

5. Chadha, S. 2015. Comparative performance of pea genotypes under organic and conventional farming conditions. Journal of Hill Agriculture. 6 (1): 29-34.

6. Chadha, S, Rameshwar, Saini, JP and Sharma, S. 2013. Performance of different varieties of pea (Pisum sativum L.) under organic farming condition in mid Himalayas. Indian Journal of Agriculture and Food Science 4 (7): 733-738.

7. Futuless, $K N$ and Bake, I D. 2010. Evaluation of yield and yield attributes of some cowpea [Vigna unguiculata (L.) Walp] varieties in Northern Guinea Savanna. Journal of Agriculture Science 6 (12): 20-25. 
8. Gomez, KA and Gomez, AA. 1984. Statistical procedures for agricultural research (2 ${ }^{\text {nd }}$ Ed.). John Wiley and sons, New York, $680 p$

9. Green, CF, Paulson, GA and Ivins, JD. 1985. Time of sowing and the development of winter wheat. Journal of Agricultural Sciences 105: 217-221.

10. Kalloo, G, Rai, M, Singh, J, Varma, A, Kumar, R, Rai, G K and Vishwanath 2005. Morphological and biological variability in vegetable pea (Pisum sativum L.). Vegetable Science 32 (1): 19-23.

11. Khan, TN, Ramzan, A, Jillani, G. and Mahemood, T. 2013. Morphological performance of peas (Pisum Sativum L.) genotypes under rainfed condition of Potowar region. Journal of Agriculture Research 51 (1): 823-830.

12. Khichi, P, Chandan, PM., Chauhan, J., Srinivas, J. and Bhagat, M. 2016. Varietal evaluation of garden pea under semi-arid conditions of Vidharba region. International Journal of Farm Sciences 6 (1): 20-24.

13. Moniruzzaman, M., Rahman, S M L, Kibria, M G, Rahman, M A and Kaisar, M O. 2007. Performances of vegetable french bean as influenced by varieties and sowing dates in rabi season. International Journal of Sustainable Crop Production 2(5):69-73.

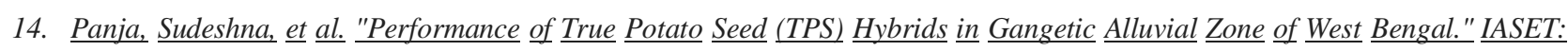

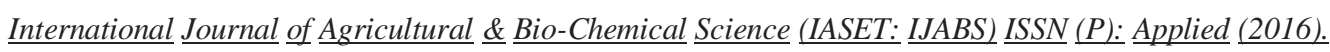

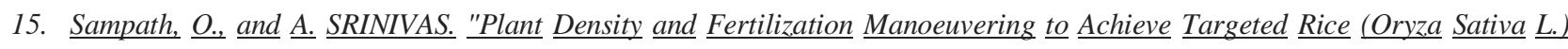

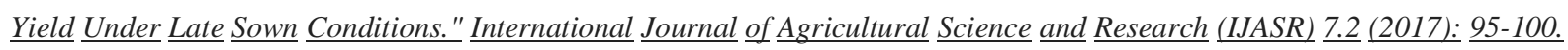

16. Sampath, $\underline{O .}$ et $\underline{\text { al. }} \underline{\text { "Effect }} \underline{\text { of plant density }} \underline{\text { and fertilizer levels }} \underline{\text { on growth parameters }} \underline{\text { of }} \underline{\text { rice }} \underline{\text { varieties }} \underline{\text { under }} \underline{\text { late }} \underline{\text { sown }}$

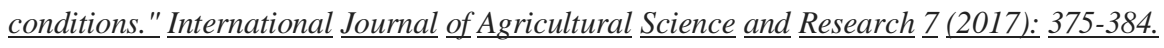

17. Salter, P J. 1963. The effect of wet or dry soil conditions at different growth stages on the components of yield of a pea crop. Journal of Horticultural Sciences 38: 321-324.

18. Sharma SK. 2002. Effect of sowing time and spacing levels on seed production of pea cultivar Arkel. Seed Research 30(1): 8891.

19. Sharma, Akhilesh, Sharma, Munish, Sharma, K C, Singh, Yudhvir, Sharma, R P and Sharma, GD. 2014. Standardization of sowing date and cultivars for seed production of garden pea (Pisum sativum var. hortense L.) Under north western Himalayas, Legume Research 37 (3): 287-293.

20. Siddique, AB, Wright, D and Ali, S M. 2002. Effects of sowing dates on the phenology, seed yield and yield components of peas. Journal of Biological Sciences 2(5): 300-303.

21. Singh, R and Singh, PM. 2011. Effect of sowing date and varieties on yield and quality of garden pea seed. Vegetable Science 38 (2): 184-187.

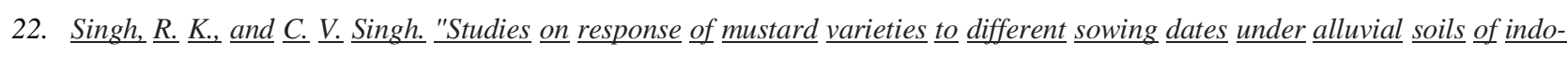

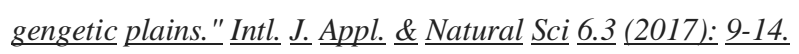

23. Sirwaiya, Sonam, Kushwah, S S, Bain, Ram Pratap and Mandale Poonam 2018. Study of combined effect of sowing dates and varieties on growth attributes in garden Pea (Pisum sativum L.). The Pharma Innovation Journal 7(5): 709-711 
